In der Rubrik „Literatur kompakt" werden die wichtigsten Originalarbeiten aus der internationalen Fachliteratur referiert.

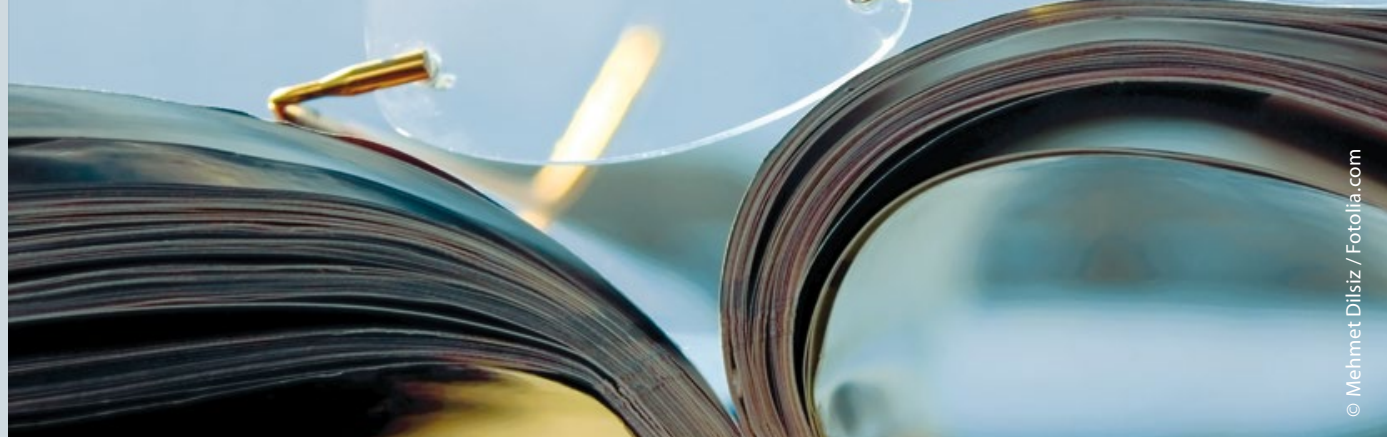

\section{Wenn Brustkrebs auf die Nerven geht}

\section{Therapiebedingte neurologische Komplikationen treten nach einer Brustkrebsdiagnose häufig auf: Fast jede zweite Frau ist betroffen, wie eine portugiesische Studie zeigt.}

A n einer prospektiven Kohortenstudie zu den neurologischen Folgen der Brustkrebstherapie waren 506 Frauen mit einem Durchschnittsalter von 55 Jahren beteiligt. Mehr als $80 \%$ hatten Karzinome im Stadium II oder darunter. 6,9\% der Frauen erhielten eine neoadjuvante Chemotherapie. Etwa die Hälfte der Studienteilnehmerinnen wurde brusterhaltend operiert, die andere Hälfte unterzog sich einer Mastektomie. Bei rund jeder dritten Patientin wurde eine axilläre Lymphknotendissektion vorgenommen. Für die meisten Frauen schloss sich eine adjuvante Therapie an in Form einer endokrinen (83,9\%), Strahlen-
(73,0\%) oder Chemotherapie (52,5\%). Letztere bestand meist aus drei Zyklen mit 5-Fluorouracil, Epirubicin und Cyclophosphamid, gefolgt von drei Zyklen Docetaxel. 71,6\% der Frauen mit Chemotherapie wurden nach einem Taxan-basierten Schema behandelt. Vor Therapiebeginn waren die Patientinnen neurologisch weitgehend unauffällig gewesen. Am häufigsten hatte eine Migräne vorgelegen (8,3\%). Doch im Verlauf des ersten Nachbeobachtungsjahres hatte fast jede Zweite (48,8\%) neurologische Beschwerden. Es dominierten neuropathische Schmerzen (30,8\%), vor allem im Gebiet des chirurgischen Eingriffs. Dabei er- reichte der mittlere Schmerzgrad einen Wert von 4,6 auf einer Skala von 1 bis 10 . Eine durch die Chemotherapie induzierte periphere Neuropathie hatten $16,8 \%$ aller chemotherapierten Frauen. Jede zehnte Patientin hatte sowohl Schmerzen als auch eine periphere Neuropathie. Relativ häufig waren auch Phantomschmerzen der Brust (16,6\%). Ein kognitiver Abbau war bei $8,1 \%$ der Frauen festzustellen.

Fazit: Neurologische Komplikationen seien ein häufiger Nebeneffekt der Brustkrebstherapie, schlussfolgern die Studienautoren. Es sei wichtig, diese Nebenwirkungen exakt zu diagnostizieren und zu behandeln, um die Krankheitslast für die betroffenen Frauen zu reduzieren.

Dr. Robert Bublak

Pereira S et al. Neurological complications of breast cancer: A prospective cohort study. Breast 2015, online 18. Juni; doi: 10.1016/j. breast.2015.05.006

käufliche, pflanzliche sowie Nahrungsergänzungsmittel. Die Zahl der Arzneien der Studienteilnehmer lag im Durchschnitt bei 9,23. Die Prävalenz von Polypharmazie, exzessiver Polypharmazie und PIM betrug 41, 43 bzw. 51\%. Es gab 173 Fälle von PIM und zwar bei 40, 38 bzw. $21 \%$ der Patienten. Mit PIM war dabei die Polypharmazie ebenso assoziiert wie eine erhöhte Komorbidität. Um die Behandlung von älteren Tumorpatienten zu verbessern, sollte ihr Medikamentengebrauch professionell analysiert werden.

\section{Christina Berndt}

Nightingale G et al. Evaluation of a pharmacistled medication assessment used to identify prevalence of and associations with polypharmacy and potentially inappropriate medication use among ambulatory senior adults with cancer. J Clin Oncol. 2015;33(13):1453-9 